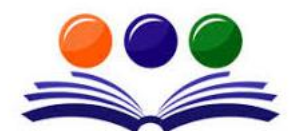

GRAND ACADEMIC PORTAL RESEARCH JOURNALS

\title{
A GLOBAL JOURNAL OF SOCIAL SCIENCES
}

( ISSN - 2581-5830 )

Impact Factor: SJIF - 4.998, IIFS - 4.375

\section{INDEX}

\begin{tabular}{|c|c|c|c|}
\hline $\begin{array}{l}\text { SR. } \\
\text { NO. }\end{array}$ & TITLE OF PAPER & AUTHOR NAME & $\begin{array}{l}\text { PAGE } \\
\text { NO. }\end{array}$ \\
\hline 1 & $\begin{array}{l}\text { A CAUSAL RELATIONSHIP BETWEEN PUBLIC } \\
\text { EXPENDITURE EDUCATION AND HUMAN } \\
\text { DEVELOPMENT: ANALYSIS OF INDIAN STATES }\end{array}$ & $\begin{array}{l}\text { Manisha Kushwaha } \\
\text { Dr. Radha Tiwari }\end{array}$ & 1-7 \\
\hline 2 & $\begin{array}{l}\text { AN ANALYSIS OF CONSUMER BUYING BEHAVIOUR } \\
\text { TOWARDS ONLINE SHOPPING: A CASE STUDY OF } \\
\text { CUTTACK CITY }\end{array}$ & $\begin{array}{l}\text { Prangyan Pradeepa } \\
\text { Mohanty, } \\
\text { Dr Biswa Mohana Jena }\end{array}$ & 8-17 \\
\hline 3 & $\begin{array}{l}\text { SIGNIFICANT OF CORPORATE SOCIAL } \\
\text { RESPONSIBILITY IN INDIA }\end{array}$ & $\begin{array}{l}\text { Krushnavadan Parmar, } \\
\text { Dr. Prashant M. Joshi }\end{array}$ & $18-23$ \\
\hline 4 & $\begin{array}{l}\text { A STUDY ON CSR PRACTICES OF SELECTED INDIAN } \\
\text { COMPANIES AND THEIR CONTRIBUTION TOWARDS } \\
\text { HEALTHCARE }\end{array}$ & $\begin{array}{l}\text { Krushnavadan Parmar, } \\
\text { Dr. Prashant M. Joshi }\end{array}$ & $24-27$ \\
\hline 5 & $\begin{array}{l}\text { A REVIEW ON THE PRACTICABILITY OF FINANCIAL } \\
\text { REPORTING AND STOCK MARKETS WITH } \\
\text { REFERENCE TO STATISTICS }\end{array}$ & Aditya Prasad Sahoo & $28-31$ \\
\hline 6 & $\begin{array}{l}\text { HEALTH AND ORGANIZATIONAL PERFORMANCE IN } \\
\text { A POST-PANDEMIC WORLD }\end{array}$ & Marcel Daane & $32-34$ \\
\hline 7 & $\begin{array}{l}\text { A STUDY ON INDIVDUALS BHEAVIOUR TOWARDS } \\
\text { E-BANKING AND M-BANKING IN BHUBANESWAR } \\
\text { CITY }\end{array}$ & $\begin{array}{l}\text { Aditya Prasad Sahoo, } \\
\text { Gayatri Pati }\end{array}$ & $35-38$ \\
\hline 8 & LEADERSHIP DURING TOUGH TIMES & Hans-Harry Bittner & $39-43$ \\
\hline 9 & $\begin{array}{l}\text { A STUDY OF MUTUAL FUND AWARENESS IN } \\
\text { GANDHINAGAR CITY }\end{array}$ & Ca Jyoti J Patel & $44-54$ \\
\hline 10 & $\begin{array}{l}\text { ETHICS OF AFRICAN'S TRADITIONAL CULTURE IN } \\
\text { CHINUA ACHEBE'S THINGS FALL APART : A STUDY }\end{array}$ & V. Sathya & $55-59$ \\
\hline 11 & $\begin{array}{l}\text { ANALYZING THE ROLE OF SOCIAL MEDIA IN } \\
\text { INVESTMENT DECISION WITH SPECIAL REFERENCE } \\
\text { TO SOUTH GUJARAT }\end{array}$ & $\begin{array}{l}\text { Ekta Ashokkumar Mistri, } \\
\text { Dr. Gurudutta P. Japee }\end{array}$ & $60-64$ \\
\hline 12 & $\begin{array}{l}\text { A COMPARATIVE STUDY ON MANAGING RISK WITH } \\
\text { RESPECT TO MANUFACTURING UNITS }\end{array}$ & Prof. Shimoni Trivedi & 65-68 \\
\hline 13 & $\begin{array}{l}\text { RELATION BETWEEN EDUCATION AND TOURISTS' } \\
\text { PERCEPTION ABOUT PILGRIMAGE TOURISM - A } \\
\text { STUDY WITH REFERENCE TO NORTH GUJARAT }\end{array}$ & $\begin{array}{l}\text { Ms. Rajpriya Laxmichand } \\
\text { Patel }\end{array}$ & $69-74$ \\
\hline 14 & $\begin{array}{l}\text { EFFECT OF DRAMATIZED TELEVISION MEDIA ON } \\
\text { MENTAL IMAGINATION OF URBAN ADULT }\end{array}$ & Smita Ghosh & $75-81$ \\
\hline 15 & $\begin{array}{l}\text { NESTLE INDIA LIMITED: GST ANTI-PROFITEERING } \\
\text { COURT CASE }\end{array}$ & $\begin{array}{l}\text { Reetu Murlidhar } \\
\text { Tanwani, } \\
\text { Dr. Mahendra. H. } \\
\text { Maisuria }\end{array}$ & 82-86 \\
\hline 16 & $\begin{array}{l}\text { ZERO BASED BUDGETING BASED ON PUBLIC } \\
\text { ENTERPRISES IN INDIA }\end{array}$ & $\begin{array}{l}\text { Ghanchi } \\
\text { Mahammadhushen } \\
\text { Kasambhai }\end{array}$ & $87-94$ \\
\hline 17 & $\begin{array}{l}\text { “A STUDY OF THE PATTERN AND DETERMINANTS } \\
\text { OF AGRICULTURAL DEVELOPMENT IN THE STATE } \\
\text { OF GUJARAT" }\end{array}$ & Dr. Geetanjali Chauhan & $95-104$ \\
\hline 18 & $\begin{array}{l}\text { PRACTICING CORPORATE SOCIAL RESPONSIBILITY } \\
\text { AMIDST COVID- } 19\end{array}$ & Payal Shastri & 105-112 \\
\hline 19 & $\begin{array}{l}\text { HISTORICAL IMPACT IN THOMAS HARDY AND R.K. } \\
\text { NARAYAN NOVELS }\end{array}$ & V. Sathya & 113-115 \\
\hline
\end{tabular}

